\title{
Incidencia en el Ahuellamiento y Propiedades Mecánicas de una Mezcla Asfáltica por la Adición de Desperdicio de Llanta Usada
}

* M.Sc. Jefe del Área de Geotecnia - Profesor Facultad de Ingeniería - Universidad Militar Mueva Granada - Bogotá Colombia.

e-mail: oreyes@umng.edu.co

** M.Sc. Profesor Facultad de Ingeniería - Universidad Militar Nueva Granada - Bogotá, Colombia.

e-mail:jcamacho@umng.edu.co

Fecha de recepción: Septiembre 30 de 2004

Fecha de aprobación: Diciembre 17 de 2004

\author{
Oscar Javier Reyes Ortíz* \\ Javier Fernando Camacho Tauta**
}

\section{RESUMEN}

El propósito de esta investigación consistió en determinar el efecto en el ahuellamiento de una mezcla asfáltica cerrada, al introducirle desperdicio de llantas usadas como reemplazo de material. El estudio inició con la caracterización de los materiales granulares, el asfalto y el desperdicio de llanta, seguido con la determinación del porcentaje óptimo de asfalto mediante el ensayo Marshall y concluyendo con la ejecución del ensayo de ahuellamiento con la adición de llanta usada como reemplazo de material granular. El desperdicio de llanta fue introducido en tres formas geométricas diferentes, la primera como polvo, la segunda 
como fibras de longitud de 1.5 centímetros y la última como una combinación equivalente de las dos anteriores. Entre los resultados obtenidos se resalta que la deformación descendió hasta un $13 \%$ para el empleo de polvo de llanta y de $23 \%$ para fibras de llanta.

Palabras Clave: Ensayo Marshall, ahuellamiento, llanta usada, mezcla asfáltica cerrada.

\section{ABSTRACT}

The propose of this research was to determine the rutting effect in the proprieties of asphalt mixture, by inserting wasted tires as a substitution of granular material. The study started with a description of the properties of granular material, asphalt and wasted tires, followed by the result of the ideal percentage obtained in the Marshall test. It concluded with the Marshall Tests performace and the rutting adding wasted tires as a substitute of granular material. Waste tires were introduced in three different geometrical shapes; one of them, powder, the second one fibers of 1.5 centimeters long and the last one, a combination of powder and fibers. The results obtained showed that deformation decreased by $13 \%$ when using wasted tires powder and by $23 \%$ when using tire fibers.

Key Words: Marshall test, rutting, used tire, asphalt mixture close.

\section{INTRODUCCIÓN}

El ahuellamiento es una falla estructural característica de los pavimentos flexibles (Figura 1), que corresponde a una deformación permanente en la estructura y la cual es generada especialmente por un exceso en el tráfico, cargas elevadas por eje, malos procesos constructivos y altas temperaturas de servicio.

Las deformaciones que se presentan en una estructura de pavimento están compuestas por las elásticas y las plásticas. (Ref [1] Thenoux
Guillermo,2001). Las primeras se recuperan luego de que la carga impuesta en la carpeta es retirada, mientras que las segundas se acumulan y generan deformación permanente (Figura 2).

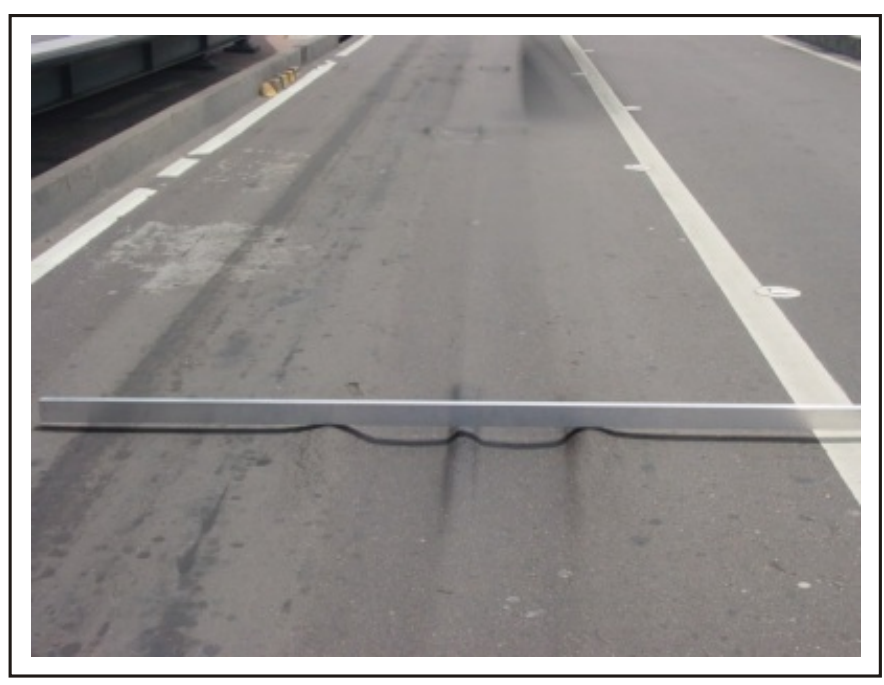

Figura 1. Fenómeno del ahuellamiento. Vía Calle 80 Bogotá Colombia

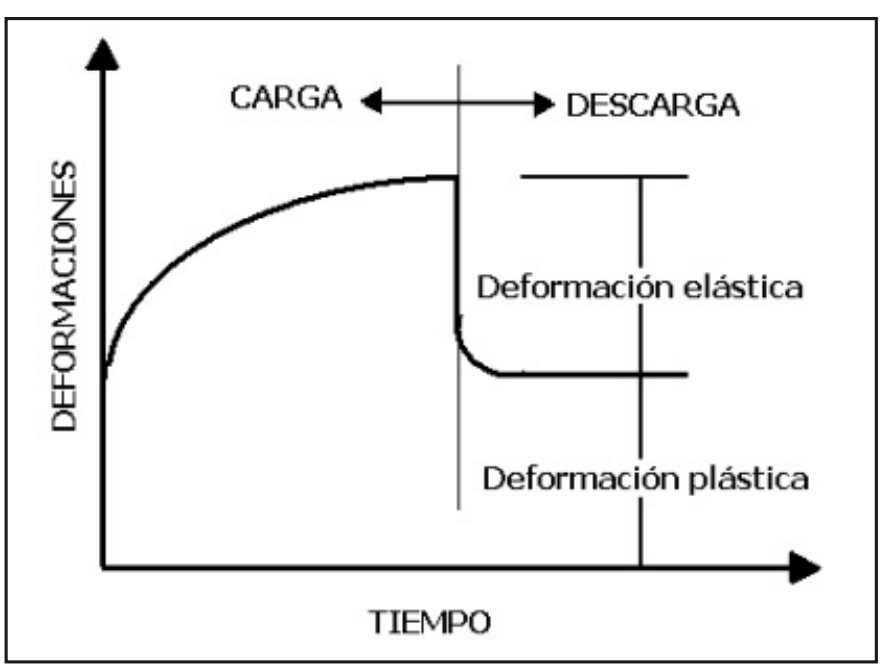

Figura 2. Comportamiento de materiales viscoelásticos

Las llantas están compuestas especialmente por fibras de acero y polímeros del tipo caucho natural (MR), caucho polisopreno (IR), caucho butadieno de estireno (SBR) o caucho polibutadieno (BR). Estos polímeros tienen propiedades térmicas, mecánicas y químicas, 
que al ser sometidos a las cargas excesivas, inclemencias del clima y agentes químicos, no presentan alteraciones significativas en sus propiedades mecánicas, lo cual hace que sea una alternativa como reemplazo de material granular en la construcción de estructuras de pavimento. Múltiples compañías han patentado productos industrializados a base de polímeros para mejorar las propiedades mecánicas y dinámicas de mezclas asfálticas, sin embargo debido a sus costos elevados poco son empleados en países en vía de desarrollo como el nuestro. Actualmente diferentes centros de investigación adelantan averiguaciones conducentes a reemplazar materiales granulares por desperdicios plásticos, basándose en los principios de los polímeros industrializados, prueba de ello son estudios como el "Fatigue behavior of a pavement foundation with recycle agregate and waste HPDE strips" (Ref [2] Sobhan, Únale, 2003), "Evaluación de las propiedades asfalto-caucho para utilización en pavimentación asfáltica" (Ref [3] Ola, Sandra, 2000) y "Use of plastic for porous asphalt mixture design" (Ref [4]Reyes, Oscar 2003) entre otros.

De otra parte, es importante resaltar, que estos productos de desperdicio plástico debido a sus grandes periodos de degradación (100 a 1000 años) (Ref [5] Unión temporal Fichtner-Cydep, 2000), generan contaminación ambiental en las cuencas hidrográficas y en terrenos de disposición de basuras.

El propósito principal de esta investigación se centró en el efecto que tiene adicionar en una mezcla asfáltica cerrada, con granulometría 0/10 y asfalto con penetración 60/70, desperdicio de llanta usada en forma de polvo, fibra y combinación de éstas en el fenómeno del ahuellamiento y a su vez contribuir en un proceso de descontaminación ambiental al emplear estos materiales en la construcción de las vías.

\section{METODOLOGÍA}

En la Figura 3 se presenta el diagrama que describe la metodología empleada para el desarrollo de este proyecto, que posteriormente se describirá en detalle.

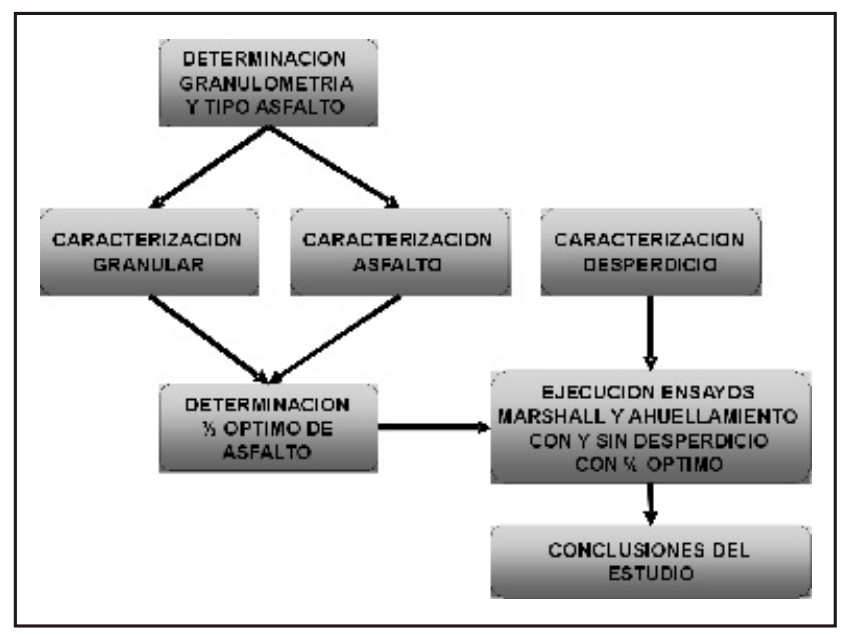

Figura 3. Diagrama de la Metodología de Investigación

\subsection{Determinación granulometría y tipo de asfalto:}

La granulometría que se empleó en la investigación corresponde a la denominada 0/10 (Figura M. 4) y es la empleada en la construcción de pavimentos en la ciudad de Bogota, según el Reglamento Técnico Vial (Ref [6] IDU-UMIAMDES, 2002). El tipo de asfalto que se utilizó fue el producido por la refinería de Barrancabermeja.

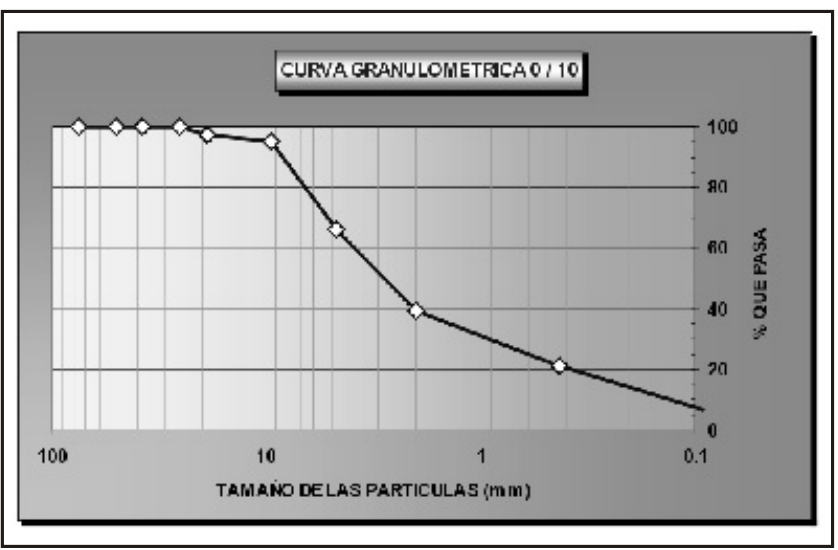

Figura 4. Curva granulométrica 0/10 


\subsection{Caracterización del granular:}

El material granular se caracterizó realizándole los ensayos de laboratorio que aparecen en la Tabla 1

Tabla 1. Ensayos caracterización de material granular

\begin{tabular}{|c|c|c|}
\hline ENSAYO & NORMA & RESULTADO \\
\hline Absorción agregado grueso & ASTM C 127 & $3.34 \%$ \\
\hline Peso Específico aparente & ASTM C 127 & 2.39 \\
\hline Absorción agregado fino & ASTM C128 & $1.77 \%$ \\
\hline Peso Específico aparente & ASTM C 128 & 2.50 \\
\hline Desgaste agregados & ASTM C 535 & $25.6 \%$ \\
\hline
\end{tabular}

\subsection{Caracterización del asfalto:}

El asfalto empleado en la investigación es el denominado de Barrancabermeja y posee las características de la Tabla 2.

\subsection{Caracterización del desperdicio plástico:}

Para la investigación se empleó material de las
Tabla 2. Ensayos caracterización del asfalto

\begin{tabular}{|l|c|c|}
\hline \multicolumn{1}{|c|}{ ENSAYO } & NORMA & RESULTADO \\
\hline Penetración & ASTMD5-97 & $67 / 70$ \\
\hline Ductilidad & ASTMD113-99 & $125 \mathrm{~cm}$ \\
\hline Viscosidad & ASTMD2170-95 & 1500 \\
\hline Ablandamiento & ASTMD36-95 & $45^{\circ} \mathrm{C}$ \\
\hline $\begin{array}{l}\text { Punto de Ilama e } \\
\text { ignición }\end{array}$ & ASTMD3143-98 & $215^{\circ} \mathrm{CY} 22^{\circ} \mathrm{C}$ \\
\hline
\end{tabular}

llantas usadas que desecha el parque automotor de la ciudad de Bogotá, las cuales están compuestas por elastómeros, con densidad de $0.87 \mathrm{~kg} / \mathrm{cm}^{3}$. El desperdicio se utilizó de tres formas geométricas diferentes. El primero como material molido y cuyo tamaño es pasa tamiz $M$. 40. El segundo en forma de tiras de 1 milímetro de espesor por 15 milímetros aproximadamente de longitud y el último una combinación de 50\% del primero y $50 \%$ del segundo. En las Figuras 5 y $5 \mathrm{~A}$ se puede observar la forma del material.

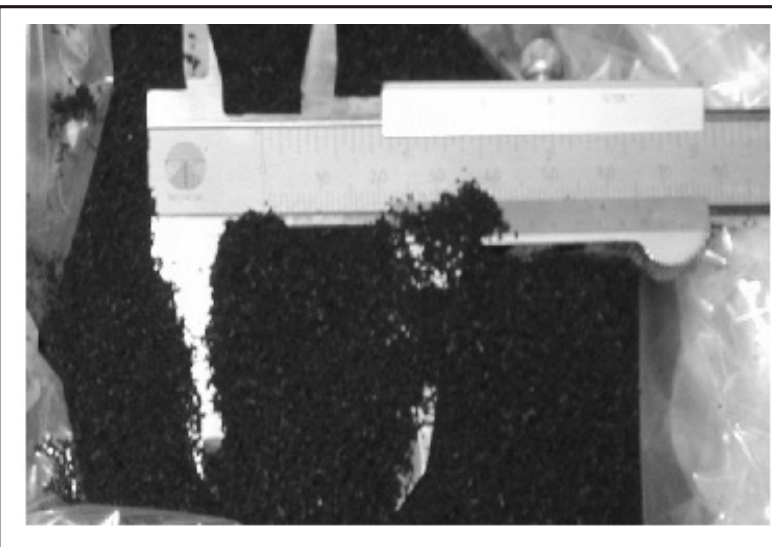

Figura 5. Llanta en polvo

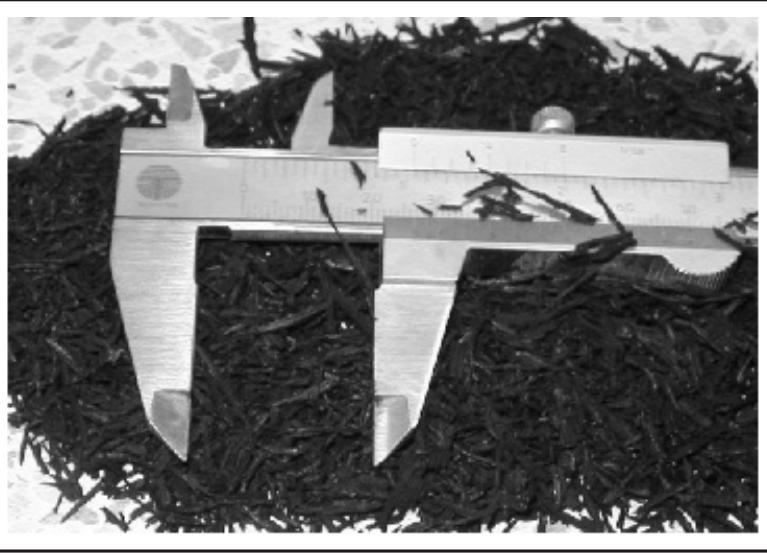

Figura 5A. Llanta en fibras

\section{ANÁLISIS DE LOS RESULTADOS}

El porcentaje óptimo de asfalto determinado con el ensayo Marshall fue de $6.5 \%$. con este porcentaje se construyeron muestras Marshall con nivel de energía a 50 y 75 golpes por cara y briquetas de ahuellamiento, a las cuales se les adicionó desperdicio de llanta entre 0.15 y $0.75 \%$ con respecto al peso del granular, con intervalos de $0.15 \%$ y en las tres formas descritas en la caracterización del desperdicio plástico.

Los resultados obtenidos para los ensayos Marshall con y sin adición de desperdicio, se observan en las Figuras de la 6 a la 10. 


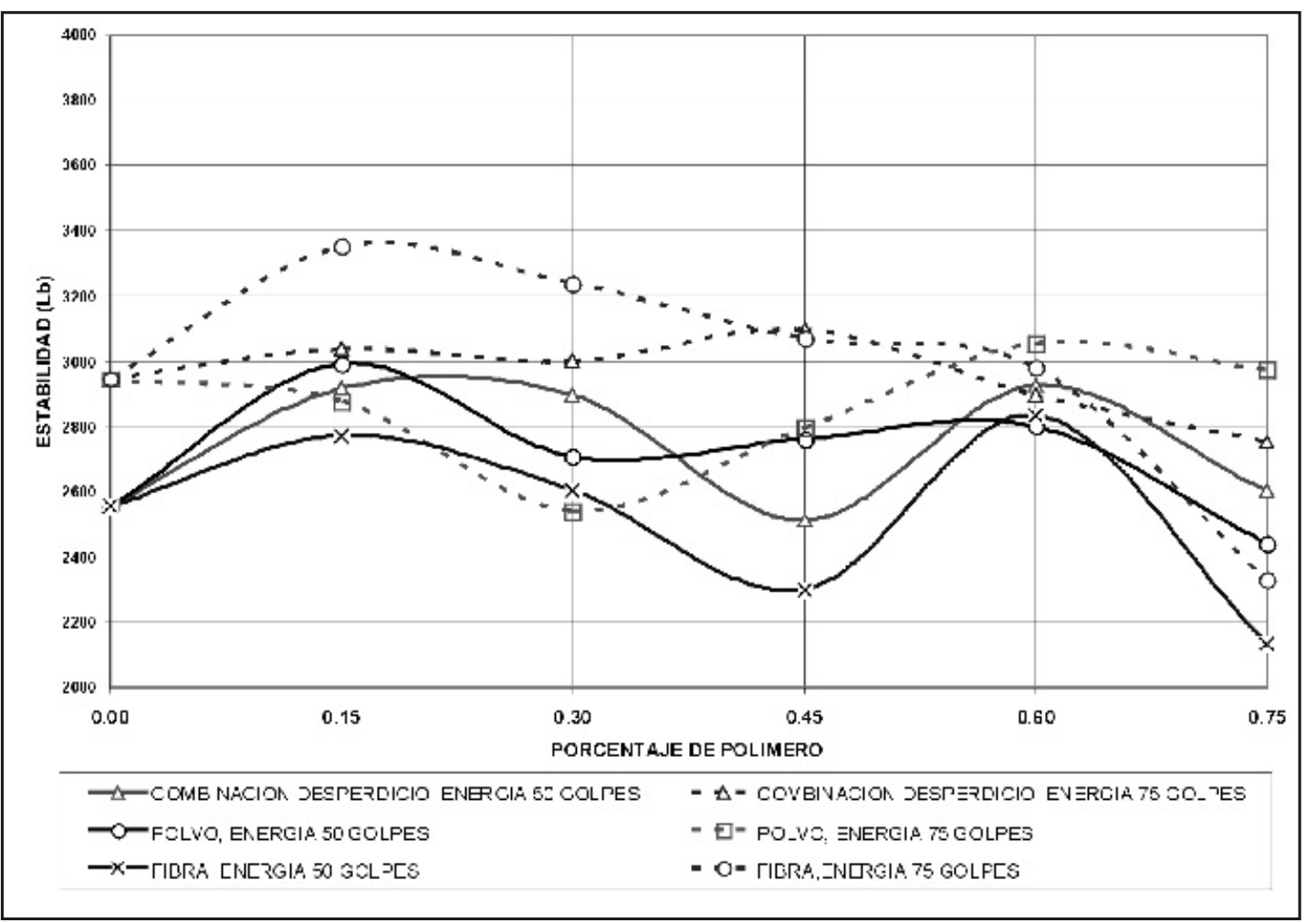

Figura 6. Variación de la estabilidad con respecto al porcentaje de desperdicio

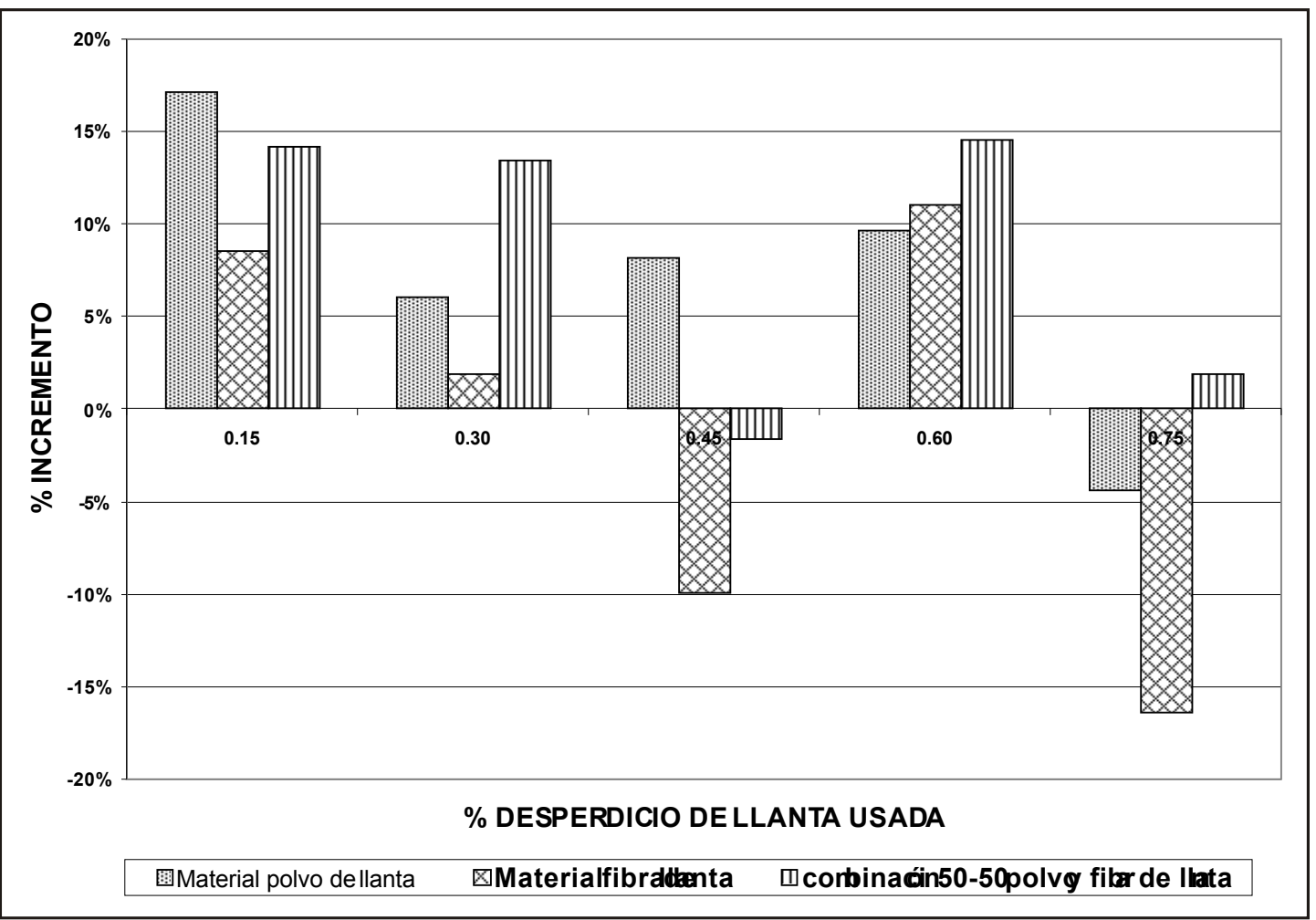

Figura 7. Incremento de la estabilidad con respecto a la adición de desperdicio de llanta usada para un nivel de energía de compactación de 50 golpes por cara 
De la Figura M. 7, se observa que para la adición de $0.15,0.3$ y $0.6 \%$, se presenta un incremento en la estabilidad de las briquetas compactadas con 50 golpes y sin importar la forma del desperdicio. Dicho incremento alcanzó su valor máximo (17\%) para un porcentaje del 0.15.
De la Figura M. 8, se observa que para la adición de fibras y nivel de energía de 75 golpes, los incrementos se presentan para la adición de desperdicio entre 0.15 y $0.6 \%$, siendo el valor máximo (14\%) para la adición del 0.15\%. Es importante resaltar que al adicionar el desperdicio en forma de polvo, se genera un descenso en la estabilidad para los valores entre 0.15 y $0.45 \%$.

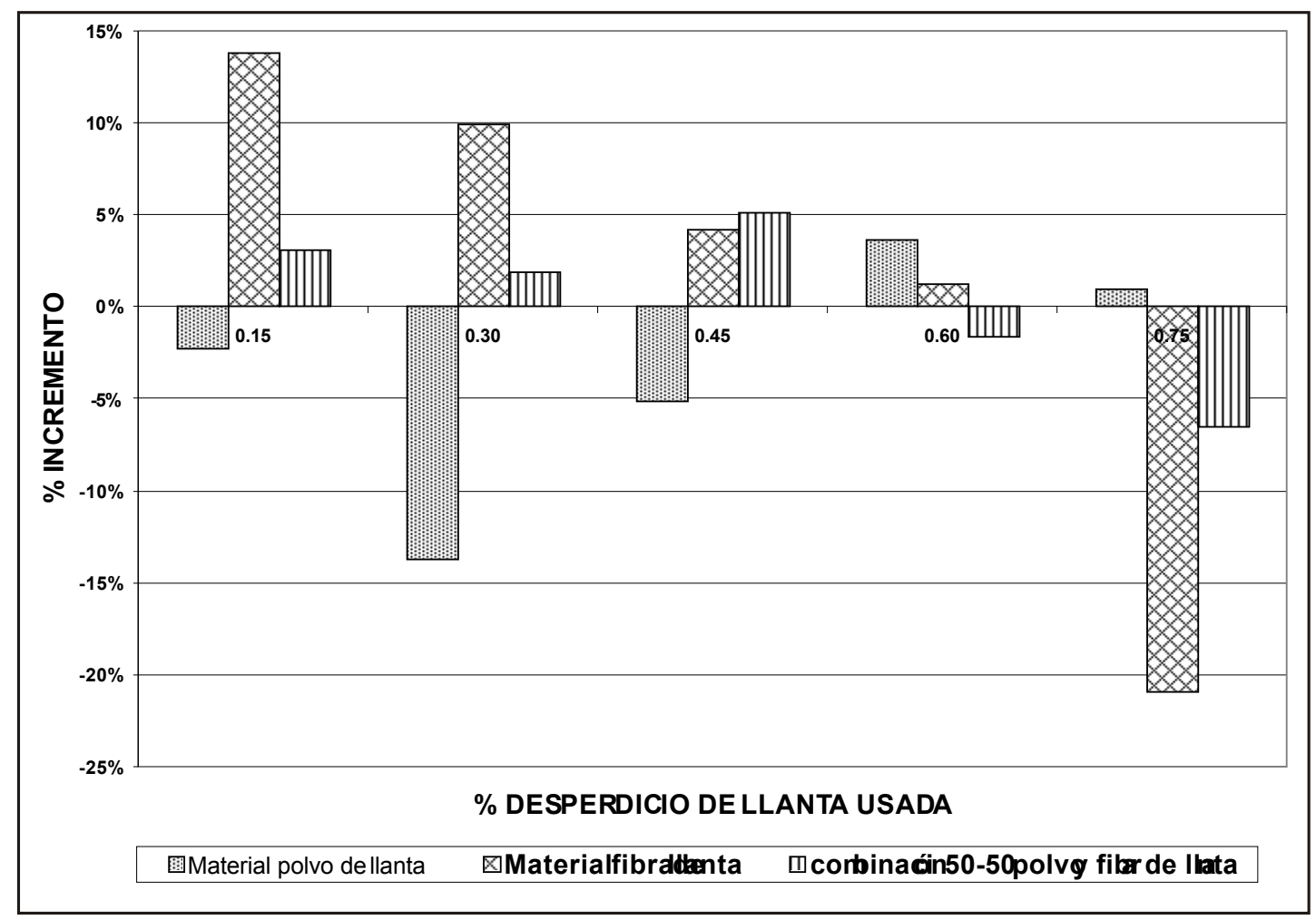

Figura 8. Incremento de la estabilidad con respecto a la adición de desperdicio de llanta usada para el nivel de energía de compactación de 75 golpes por cara

De la Figura M. 9, se puede concluir que al adicionar desperdicio en la mezcla asfáltica se reduce la densidad sin importar el porcentaje y la forma del mismo.

De la Figura M. 10, se concluye que al adicionar desperdicio a la mezcla, sin importar el porcentaje y su forma, la deformación se encuentra dentro del rango permisible.

Los resultados obtenidos de los ensayos de ahuellamiento, se observan en la Figura M. 11 para el empleo de desperdicio de llanta en forma de polvo, en la Figura N. 12 para el uso de fibras y en la Figura M. 13, la combinación de las dos.

Con base en la Figura M.14, se determinó que para el porcentaje de $0.45 \%$ y en la forma de fibra, la deformación debida al ahuellamiento disminuye hasta un $23 \%$ y en un $13 \%$ para el $0.6 \%$ de polvo de llanta. 


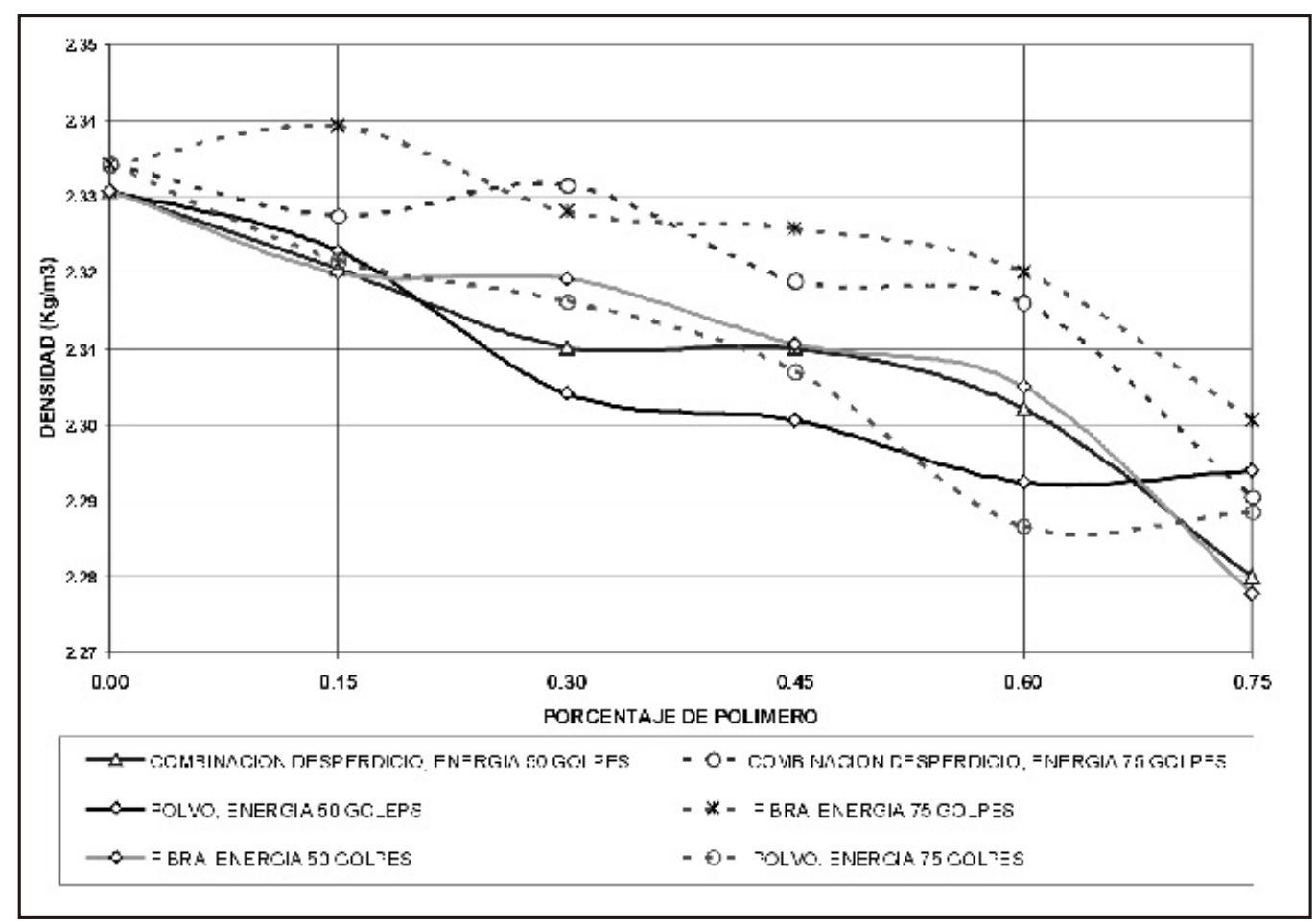

Figura 9. Variación de la densidad con respecto al porcentaje de desperdicio

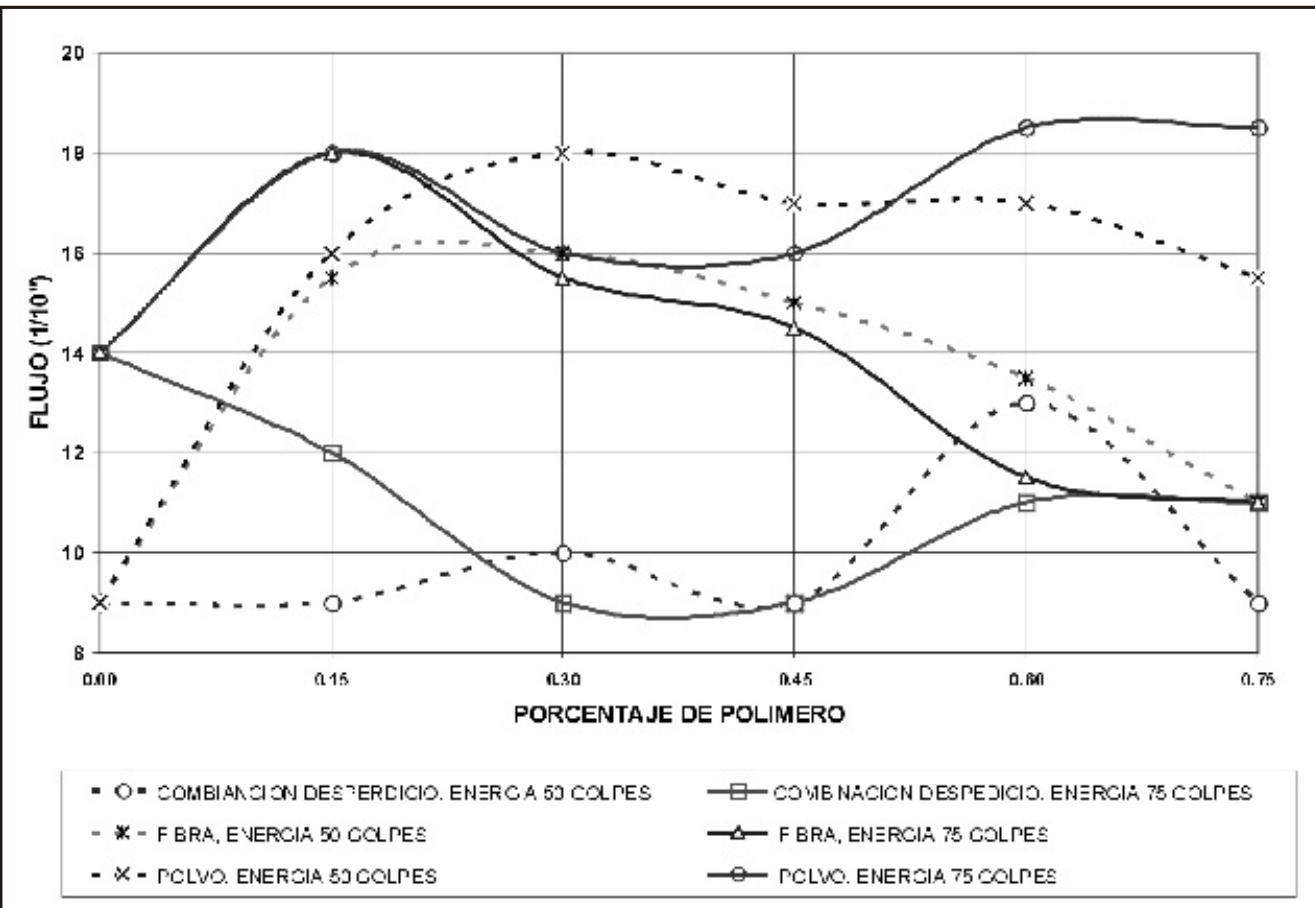

Figura 10. Variación del flujo con respecto al porcentaje de desperdicio 


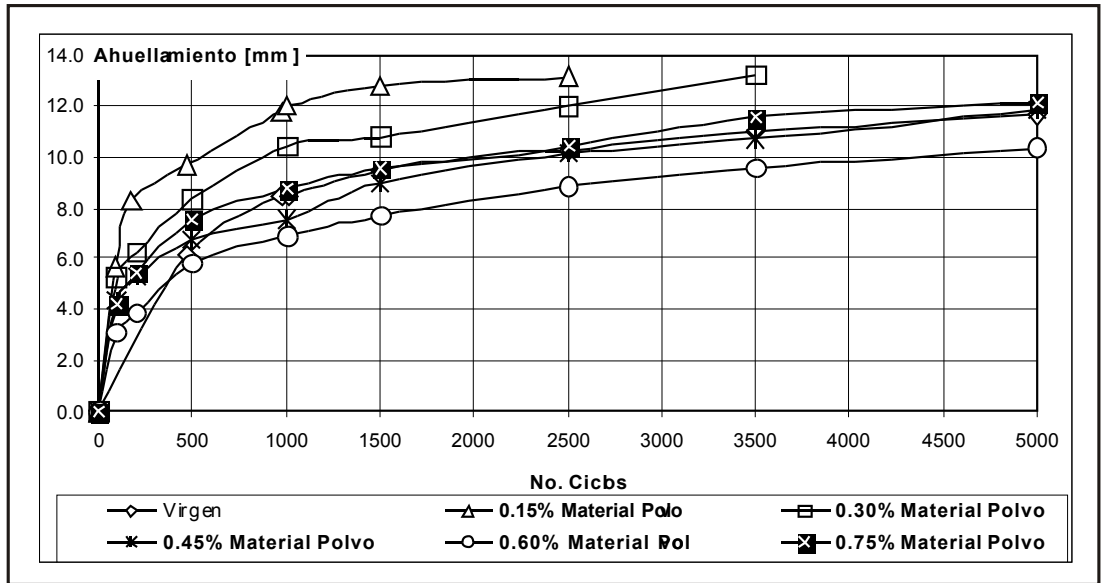

Figura 11. Variación del ahuellamiento con respecto al porcentaje de llanta usada en forma de polvo

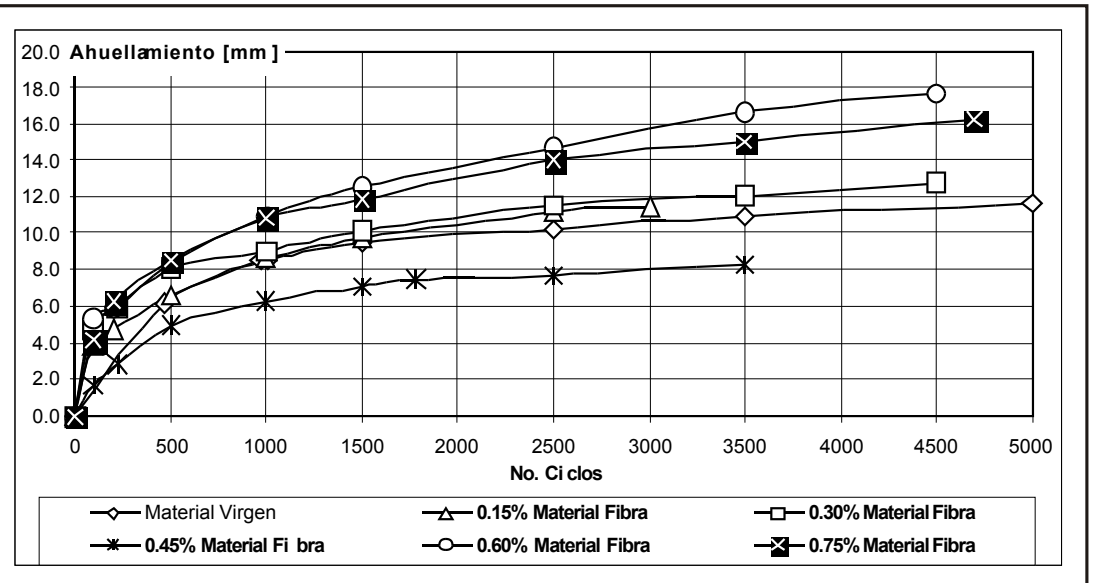

Figura 12. Variación del ahuellamiento con respecto al porcentaje de llanta usada en forma de tiras

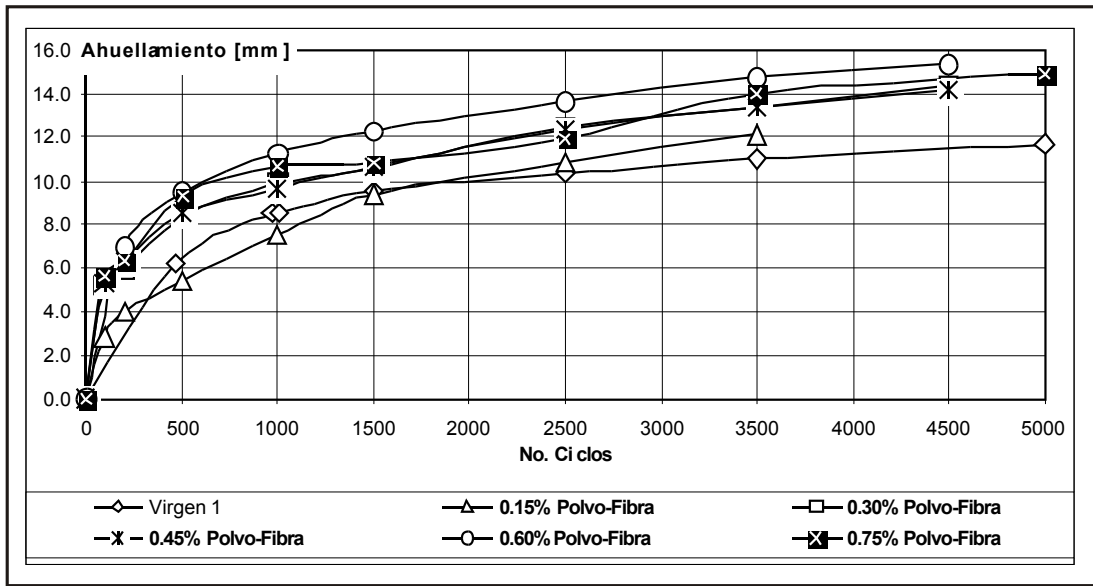

Figura 13. Variación del ahuellamiento con respecto al porcentaje de llanta usada con combinación de polvo y fibra 


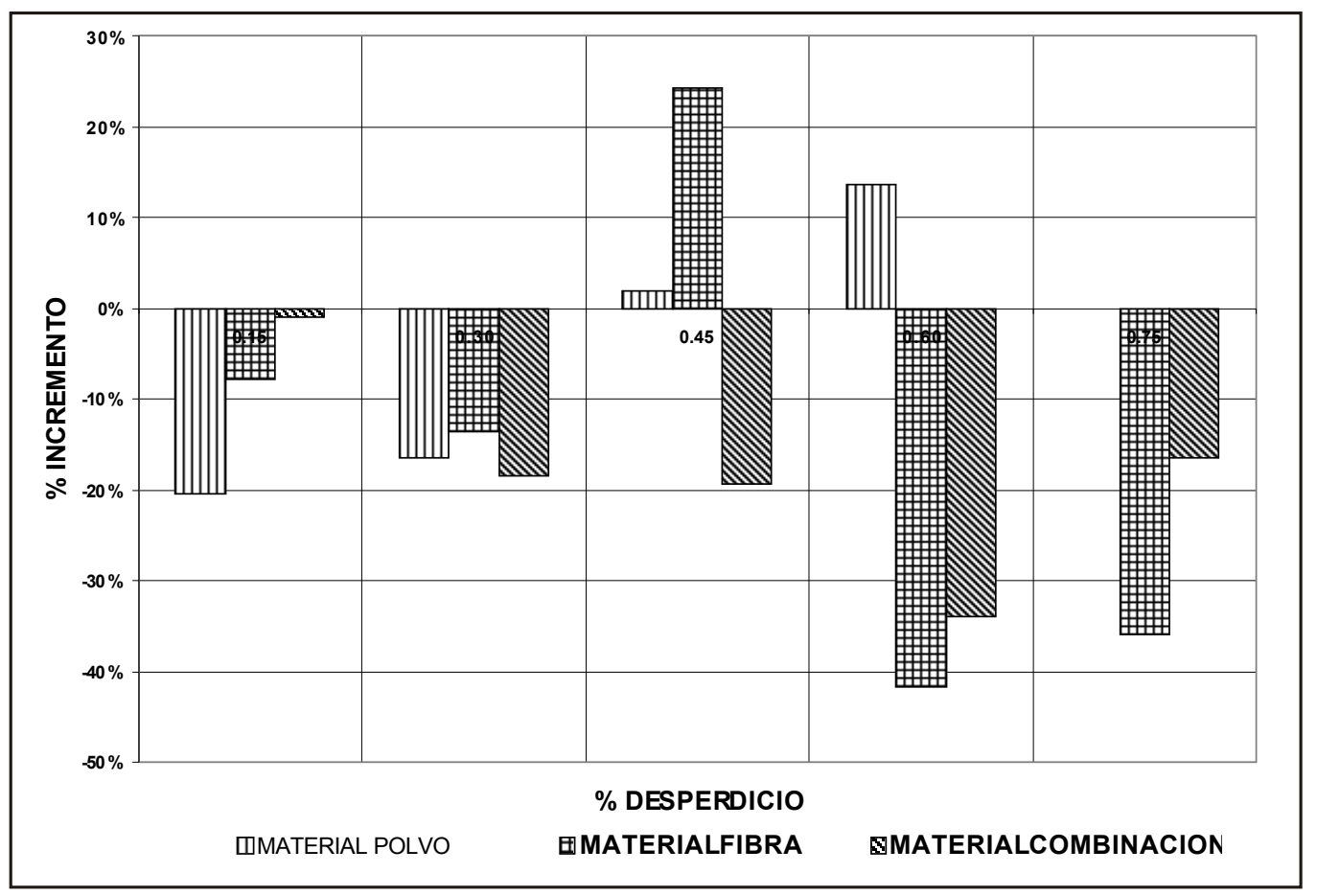

Figura 14. Efecto del desperdicio en el ahuellamiento

\section{CONCLUSIONES}

El comportamiento de la mezcla asfáltica cerrada según lo observado en el análisis de los ensayos de laboratorio, presenta un menor ahuellamiento cuando se adiciona desperdicio de llanta usada en las formas de polvo y fibra, alcanzando hasta un descenso en deformación del orden del 23\%. De otra parte, analizada la resistencia (estabilidad) para los niveles de energía de 50 golpes por cara, se incrementa al adicionar desperdicio en todas las formas para los porcentajes de $0.15 \%, 0.3 \%$ y $0.6 \%$. Para el nivel de energía de 75 golpes, los incrementos se obtuvieron para las adiciones de fibra a porcentajes de $0.15,0.3$ y $0.45 \%$. Se resalta que para la adición de polvo, existe un descenso de la estabilidad para los porcentajes de $0.15,0.3$ y $0.45 \%$.

Por último, se determinó que al mezclar el material de desperdicio en las proporciones 50\% de polvo y $50 \%$ en fibra, los resultados en los ensayos de ahuellamiento, no fueron favorables, encontrándose incrementos en la deformación hasta de un $33 \%$.

\section{AGRADECIMIENTOS}

Los autores del artículo agradecen a la Universidad Militar Mueva Granada, especialmente al proyecto IMG-2004-003, a los estudiantes que nos colaboraron en la ejecución de los ensayos y a la Universidad Javeriana.

\section{REFERENCIAS}

[1] Thenoux 2., Guillermo y otros "Análisis de casos de ahuellamiento en mezclas asfálticas chilenas". Departamento ingeniería y gestión de la construcción, pontificia universidad Católica de Chile. 2001

[2] Sobhan, Khaled and Mashnad, m. "Fatigue behavior of a pavement foundation with recycle agregate and waste HPDE strips" Journal of geotechnical and geoenvironmemtal engineering. ASCE. 2003.

[3] Ola, Sandra y otros. "Evaluación de las propiedades asfalto-caucho para utilización 
en pavimentación asfáltica". Departamento de ingeniería civil. Universidad Estatal de Maringá. DEC/UEM Brasil. IV Congreso de ingeniería de transporte. Valencia CIT 2000.

[4] Reyes Ortiz, Oscar y otros "Use of plastic for porous asphalt mixture design" Asphalt Rubber congress. Brasilia 2003.

[5] Unión temporal Fichtner-Cydep. "Segundo informe del plan maestro para el manejo integral de residuos sólidos en Santafe de Bogotá, D.C. Marzo 2000.

[6] Uniandes Idu "Reglamento técnico vial para la ejecución de obras en el sector vial de Bogotá,D.C. . 2002.

[7] American Society State Highway and transportations Officials standards "Annual book of American Society for testing and materials". 2000.

[8] Reyes, Fredy Alberto. "Diseño racional de pavimentos. Editorial CEJA. 2003.

[9] Reyes Oscar y otros. "Efecto del desperdicio plástico en las propiedades dinámicas de una mezcla asfáltica. Universidad de los Andes. Tesis de Maestria. 1998.

[10] Troncoso, Juan y Ospina Lady. "Efecto de las llantas usadas en las propiedades de una mezcla asfáltica". Universidad Militar Mueva Granada. 2004.

[11] State of California Departamento $f$ transportation. "Asphalt rubber usage guide". 2003. 